\title{
Diosmetin modulates lipogenesis and alleviates inflammatory response in nonalcoholic steatohepatitis through STAT1/CXCL10-dependent pathway
}

\author{
Nanxuan Luo ${ }^{1}$, Changqing Yang ${ }^{1}$, Yurong Zhu ${ }^{1}$, and Baoshun Zhang ${ }^{1}$ \\ ${ }^{1}$ Southwest University
}

August 5, 2020

\begin{abstract}
Background and Purpose: Diosmetin (Dios) exerted a hepatoprotective effect against nonalcoholic steatohepatitis (NASH), but few reports interpreted clearly that the alleviation of Dios against NASH were associated with the inhibition of signal transducers and activators of transcription 1 (STAT1) and macrophage chemotactic ligand 10 (CXCL10). Meanwhile, the mechanism of Dios involved with STAT1/CXCL10 mediated pathway was unknown yet. Experimental Approach: Here, high-fat diet (HFD) and palmitic acid (PA) were used to induced NASH model in mice and HepG2 cells, respectively. The liver RNA-Seq was used to reveal the key targets interfered by Dios. The impacts of Dios on the expressions of STAT1, CXCL10 and the levels of genes and proteins associated with lipogenesis and inflammation were measured by qRT-PCR and western blot assays. The STAT1 inhibitor, STAT1 overexpression plasmid, and CXCL10 siRNA were utilized to clarify the mechanism of Dios against NASH through STAT1 / CXCL10. Key Results: Dios could reduce functional parameters and morphological changes in HepG2 cells and NASH mice. Additionally, Dios alleviated the expressions of key targets STAT1 and CXCL10, and their downstream proteins involved with lipogenesis and inflammation. Interestingly, NASH was ameliorated in STAT1-inhibited mice and HepG2 cells. Unfortunately, transfecting HepG2 cells with STAT1 over-expression plasmid aggravated the development of NASH and reduced Dios-conferred hepatoprotective effect. Conclusions and Implications: The alleviation of Dios against NASH was through mediation of lipogenesis and inflammatory response via STAT1/CXCL10-dependent pathway. Therefore, Dios has good hepatoprotective effects and potentiality as a promising therapeutic agent for NASH in clinical application.
\end{abstract}

\section{Keywords}

Nonalcoholic steatohepatitis (NASH) ; Diosmetin (Dios); Signal transducers and activators of transcription 1 (STAT1); Macrophage chemotactic ligand 10 (CXCL10); Lipogenesis; Inflammation.

\section{Introduction}

Nonalcoholic fatty liver disease (NAFLD) is a continuous disease with an estimated global prevalence of 24 $\%$, encompassing a wide histological spectrum rang from steatosis to nonalcoholic steatohepatitis (NASH), advanced fibrosis, and even hepatocellular carcinoma (Lau, Zhang \& Yu, 2017; Younossi et al., 2018). The occurrence of NASH was always characterized by lipid accumulation and inflammation (Schuster et al., 2018; Tomita et al., 2016). Certain evidence indicated AMPK (AMP-activated protein kinase) was a central energy mediator in NASH, which decreased NF- $\chi \mathrm{B}$ pathway and activated mitochondrial biogenesis (Herzig \& Shaw, 2018; Liu et al., 2011; Strzyz, 2020; Wang et al., 2017). Differed from the above findings, 
STAT1 (signal transducers and activators of transcription) and CXCL10 (macrophage chemotactic ligand 10) could be regarded as potential targets for suppressing NASH in our present study. STAT1 induced the release of cytokines to initiate inflammatory response (Chmielewski et al., 2014). Blockade of STAT1 elevated the indicators of fatty acid oxidation in STAT1 ${ }^{-/-}$mice (Sisler et al., 2015). Moreover, the decrease of STAT1 inhibited inflammatory response and prevented NASH and fibrosis (Grohmann et al., 2018). Generally, transcription factor STAT1 bound to CXCL10 promoter after its phosphorylated activation and nuclear translocation, and STAT1 inhibition reduced the release of CXCL10 (Tomita et al., 2017). CXCL10 contributed to inflammatory response in NASH model and emerged as a biomarker for the clinical diagnosis of NASH (Ibrahim et al., 2016; Zhang et al., 2014). No doubt, STAT1 and CXCL10 were closely related to lipid accumulation and inflammatory response. So the interference on the regulation of STAT1 and CXCL10 was a possible effective strategy for NASH treatment.

Diosmetin (3', 5, 7-trihydroxy-4'-methoxy flavone, Dios), as a natural flavonoid (Liao et al., 2014), has a favorable effect against NASH through different mechanisms (Marcolin et al., 2012; Wu et al., 2019; Zheng et al., 2018). Recent studies demonstrated that Dios attenuated lipopolysaccharide / D-galactosamine (LPS/DGalN)-induced acute hepatic failure correlating with MAPK pathway (Yang et al., 2017), and inhibited the activation of NLRP3 inflammasome in lung injury (Liu et al., 2018). Except that, Dios activated AMPK to alleviate the liver pathological changes and release of inflammatory factors in HFD-fed NAFLD rats (Zheng, Guo, Zhong \& Jiang, 2018). What's more, our previous study revealed that Dios exhibited an effect on lipid metabolism by promoting fatty acid oxidation in diabetes-mice. From these results, we reasonably assumed that Dios exerted a hepatoprotective effect against NASH. However, whether STAT1 and CXCL10 mediate the inhibition of Dios on NASH, and how to take advantage of the relations between the downstream proteins of STAT1 and CXCL10 to fully elucidate the underlying mechanism, and if this mechanism is beneficial for us to provide a new strategy for NASH treatment, all of these need to be investigated. In this study, we utilized the established models that HepG2 cells and C57BL/6J mice respectively induced by palmitic acid (PA) and high-fat diet (HFD) to identify the protective effect and potential mechanism of Dios against NASH through mediation of lipogenesis and alleviation of inflammatory response.

\section{Materials and Methods}

\section{Chemicals and reagents}

Dios (Fig. S1) was from the College of Pharmaceutical Sciences, Southwest University, P. R. China. The standard diet (SD, HD005) and high-fat diet (HFD, HD001+2\% cholesterol, containing $45 \%$ fat and $2 \%$ cholesterol) were purchased from BiotechHD Co.Ltd. (Beijing, China). The aspartate aminotransferase (AST), alanine aminotransferase (ALT), total cholesterol (TC), triglyceride (TG), low-density lipoprotein cholesterol (LDL-C) and high-density lipoprotein cholesterol (HDL-C) commercial kits were obtained from Nanjing Jiancheng Bioengineering Institute (Jiangsu, China) and the triglyceride (TG) commercial kit was purchased from Applygen Technologies Inc. (Beijing, China). The Oil Red O and Nile Red staining kits were obtained slarbio (Beijing, China). Dulbecco's modified Eagle medium (DMEM) was purchased from KeyGen Biotech (Jiangsu, China), and fetal bovine serum (FBS) was obtained from Tianhang (Zhejiang, China). Palmitic acid (PA) was purchased from Sigma-Aldrich (St. Louis, MO, USA). Fludarabine (HY-B0069, STAT1 inhibitor) was purchased from MedChemExpress (NJ, USA). siRNA for CXCL10 (human) and the control siRNA (human) were purchased from Tsingke Biological Technology (Beijing, China). The STAT1 overexpression plasmid STAT1-pcDNA3.1 and the control plasmid pcDNA3.1 were obtained from Pulateze (Hunan, China). Lipo6000 transfection reagent was obtained from Beyotime Biotechnology (Shanghai, China). Antibodies against STAT1 (1:5000), CXCL10 (1:500), sterol regulatory element-binding proteins-1c (SREBP-1c) (1:1000), carbohydrate response element-binding protein (CHREBP) (1:1000), liver X receptor-alpha $(\operatorname{LXR} \alpha)(1: 1000)$, liver X receptor-beta $(\operatorname{LXR} \beta)(1: 1000)$, nuclear factor- $x \mathrm{~B}$ p65 (p65) (1:1000), phosphorylated-p65 (p-p65) (1:1000), tumor necrosis factor- $\alpha$ (TNF- $\alpha)(1: 1000)$, interleukin-6 (IL6) (1:1000), $\beta$-actin (1:2000) and Lamin B (1:2500) were obtained from Proteintech Group, Inc. (Hubei, 
China). Antibody against STAT1 ${ }^{\mathrm{Y} 701}$ (1:1000) was purchased from Affinity Biosciences (Jiangsu, China). Antibody against STAT1 ${ }^{\text {S727 }}$ (1:1000) was obtained from Beyotime Biotechnology (Shanghai, China).

\section{Animals experiments}

Animal studies are reported in compliance with the ARRIVE guidelines (Kilkenny, Browne, Cuthill, Emerson \& Altman, 2010) and with the recommendations made by the British Journal of Pharmacology (Curtis et al., 2018). All animal experiments were performed in accordance with the National Institutes of Health Guide for the Care and Use of Laboratory Animals. The animal studies were performed after receiving approval of the Institutional Animal Care and Use Committee (IACUC) in Southwest University, P.R. China (IACUC approval No. yxy202007). All the animals were housed under specific pathogen-free (SPF) conditions at a controlled temperature $\left(22-25{ }^{\circ} \mathrm{C}\right)$ and humidity $(50 \pm 5 \%)$, and alternating 12 -hour light / dark cycles. The animals used in this study were housed in individually ventilated cages with natural soft sawdust as bedding up to five per cage and maintained on a normal chow diet with clean water ad libitum.

Eight-week-old male C57BL/6J mice (18-22g) were obtained from Beijing Huafukang Biotechnology Co., Ltd. (SCXK-Jing-2019-0008, Beijing, China). Mice were fed with either SD or HFD for 14 weeks to induce NASH model. Thereafter, the HFD-fed NASH mice were randomly divided into HFD group and HFD + Dios group ( $\mathrm{n}=5$ per group) and administered intragastrically either Dios of $60 \mathrm{mg} \cdot \mathrm{kg}^{-1}$.day or saline for four weeks (Yang et al., 2017). Mice were euthanized by cervical dislocation under anesthesia, and the livers were removed by two independent individuals who were blinded to the treatments and either snap-frozen or fixed in buffered $4 \%$ formalin for RNA-Seq and followed experiments.

To explore the potential mechanism of STAT1 in the beneficial effect of Dios in NASH, another animal experiment was carried out ( Eight-week-old Male C57BL/6J, SCXK-Jing-2019-0010) that mice were injected with fludarabine (Flu, a STAT1 inhibitor, $0.8 \mathrm{mg}^{*} \mathrm{~kg}^{-1 *}$ day) by the abdominal cavity two cycles for 5 days every 2 weeks in the whole experiment, and mice were fed with either SD or HFD for 14 weeks to induce NASH. Thereafter, the HFD-fed mice were randomly divided into different groups ( $\mathrm{n}=5$ per group) including HFD, HFD + Dios, Flu-HFD, and Flu-HFD + Dios, and those mice were either administered intragastrically Dios $\left(60 \mathrm{mg}^{*} \mathrm{~kg}^{-1 *}\right.$ day) or saline for four weeks for four weeks. The diet intake of the mouse was monitored per day and the body weight was recorded weekly. After the last administration, all the mice euthanized by cervical dislocation under anesthesia, and the livers, kidneys, spleens, and abdominal fat pads were removed and weighed by two independent individuals who were blinded to the treatments. Serum was prepared by solidification and centrifugation (4 degC, $12000 \mathrm{xg}, 10 \mathrm{~min}$ ) and stored at $-80 \mathrm{deg} \mathrm{C}$ until the analysis of biochemical parameters. Liver samples were either snap-frozen or fixed in buffered $4 \%$ formalin for histological staining, hepatic triglyceride content measurement, quantitative real-time PCR, and western blot assay.

\section{Cell culture and treatment}

HepG2 cells were obtained from the Cell Bank at the Chinese Academy of Sciences (Shanghai, China) and cultured in DMEM supplemented with $10 \% \mathrm{FBS}$ and $1 \%$ penicillin/streptomycin in a $5 \% \mathrm{CO}_{2}$ humidified incubator at $37 \mathrm{degC}$. HepG2 cells were treated with different doses $(0.10,0.15,0.20,0.25,0.30,0.35$ and $0.40 \mathrm{mM}$ ) of PA for $24 \mathrm{~h}$ to induce NASH cell model. To detect the effect of Dios, HepG2 cells were treated with or without Dios under the indicated concentrations combined with PA treatment for $24 \mathrm{~h}$. To study the importance of STAT1 in Dios-mediated hepatoprotective effect, the STAT1 inhibitor fludarabine $(10 \mu \mathrm{M})$ was used to pretreat HepG2 cells for $1 \mathrm{~h}$ before Dios and PA.

\section{Transfection of overexpression plasmid and siRNA}

HepG2 cells $\left(3 \times 10^{5}\right.$ cells per well $)$ were seeded in 6 -well plates and incubated until the cells reached $70-80 \%$ confluence. Cells were then transfected with the STAT1 reporter vector STAT1-pcDNA3.1 
(Fig. S5), the control vector pcDNA3.1 and siCXCL10 (5'-GGUCUUUAGAAAAACUUGATT-3', 3'UCAAGUUUUUUCUAA-AGACCTT-5') mixed in Lipo6000 reagent according to the manufacturer's guidelines. Then, the medium was exchanged with fresh complete medium after $6 \mathrm{~h}$. After $24 \mathrm{~h}$ transfection, cells were incubated with $0.2 \mathrm{mM}$ PA in the presence or absence of Dios $(80 \mu \mathrm{M})$ for $24 \mathrm{~h}$. After treatment, cells were harvested for further experiments.

\section{RNA Sequencing (RNA-Seq)}

Total RNA isolated from the livers of C57BL/6J mice was used to construct high throughput sequencing libraries using NEBNext@ UltraTM RNA Library Prep Kit. High throughput RNA-sequencing was performed using a HiSeq 4000 instrument (Illumina) at Novogene (Beijing, China). Adaptor sequences and low-quality reads were initially filtered from the raw data. Then the remaining ones, called clean reads, were aligned to the reference genome of a mouse, using the HISAT2 v2.0.5 program. Subsequently, unigene expression was calculated as the FPKM (fragments per kilobases of exons for per million mapped reads) with featureCounts v1.5.0-p3. Differential expression genes (DEGs) of two groups were performed using the DESeq2 $\mathrm{R}$ package, and when the P-value was less than 0.05 and the $\log _{2}$ ratio was greater than 1 (two-fold change), the unigenes were considered to be differentially expressed.

\section{GO and pathway enrichment analyses of DEGs}

The enrichment analysis of GO (Gene Ontology) and KEGG (Kyoto encyclopedia of genes and genomes) pathway was performed for DEGs using David which is an online biological information database for annotation, visualization, and integrated discovery (Huang, Sherman \& Lempicki, 2009). GO consists of biological processes (BP), cellular components $(\mathrm{CC})$, and molecular functions $(\mathrm{MF})$. P-value $<0.05$ was chosen as the cut-off point for GO and KEGG analyses.

\section{PPI network, module analysis, and hub genes identification}

Protein-protein interaction (PPI) analysis of differentially expressed genes was based on the STRING database. The significant module in the PPI network was identified by molecular complex detection (MCODE). The parameters of DEGs clustering and scoring were set as follows: MCODE score [?] 4, degree cut-off $=2$, node score cut-off $=0.2$, max depth $=100$, and $\mathrm{k}$-score $=2$. Hub genes were identified using Cytohubba (a plug-in of Cytoscape software) filtered with the criterion of degrees $>10$ criteria (each node had more than 10 interactions).

\section{Biochemical assessment of serum and liver}

Serum triglyceride (TG), total cholesterol (TC), low-density lipoprotein cholesterol (LDL-C), high-density lipoprotein cholesterol (HDL-C), alanine transaminase (ALT) and aspartate aminotransferase (AST) were quantified using enzymatic assays. The content of TC and TG of liver tissues and HepG2 cells were determined with an enzymatic assay kit and the protein concentrations were determined with a BCA protein assay kit. The results were expressed as mmol per g protein $\left(\mathrm{mmol}^{*} \mathrm{~g}^{-1}\right)$. All procedures were carried out according to the manufacturer's instructions.

\section{Histological assessment}

Histologic examination of the liver was performed by hematoxylin-eosin staining. Liver samples were fixed briefly in $4 \%$ paraformaldehyde and embedded with paraffin. The samples were cut into $5 \mu \mathrm{m}$ sections, deparaffinized, rehydrated, then stained with hematoxylin-eosin or mason trichrome. Images of liver sections were captured with light microscope. The amounts of steatosis, activity, and fibrosis were scored using the Fatty Liver Inhibition of Progression (FLIP) Algorithm (Bedossa \& Consortium, 2014). The SAF score 
was defined as the total of scores for steatosis (S0-S3), activity including lobular inflammation (0-2) and hepatocellular ballooning (0-2) scores, and the fibrosis (F0-F4) score.

\section{Immunofluorescence analysis}

Liver samples embedded with paraffin were dewaxed, blocked, and then incubated with phosphorylated STAT1 $1^{\text {Tyr701 }}$ and phosphorylated STAT1 ${ }^{\text {Ser727 }}$ antibodies overnight at $4{ }^{\circ} \mathrm{C}$. Slides were washed three times with PBS and then incubated with secondary antibody labeled fluorescence for $30 \mathrm{~min}$ at $37^{\circ} \mathrm{C}$. The nuclei were counterstained with 4', 6-diamidino-2-phenylindole (DAPI) for $5 \mathrm{~min}$ at room temperature. Images were captured using fluorescent microscopy.

\section{Lipid content analysis}

Hepatic lipid accumulation was detected by Oil Red O staining. Liver sample cryosections and hepatocytes were prepared for staining with pre-warmed Oil Red $\mathrm{O}$ working solution for $20 \mathrm{~min}$, being rinsed with $60 \%$ isopropanol 3 times. And then counterstained with hematoxylin for $3 \mathrm{~min}$, gently washed with $60 \%$ isopropanol. The images were captured using light microscopy. HepG2 cells were seeded in a 24-well plate and treated with $0.2 \mathrm{mM} \mathrm{PA}$ and indicated concentrations of Dios for $24 \mathrm{~h}$. The cells were washed twice with PBS and fixed with $4 \%$ formaldehyde for 20 min and then stained with $1 \mu \mathrm{g} \cdot \mathrm{mL}^{-1}$ Nile red for $30 \mathrm{~min}$ at room temperature. Lipid-bound Nile red fluorescence was detected using fluorescence microscopy.

\section{Quantitative real-time polymerase chain reaction(qRT-PCR)}

To verify the quantification of gene expression levels, qRT-PCR was performed as previously described (Zhou et al., 2018). Total RNA was extracted from livers and HepG2 cells using Trizol reagent (Sangon Biotech Co., Ltd., Shanghai, China), and RNA was reverse-transcribed using the Fastingking RT kit (TiangenBiotech CO. LTD., Beijing, China). Quantitative real-time PCR was carried out with SYBR Premix Plus (TiangenBiotech CO. LTD., Beijing, China) according to the manufacturer's instructions, and the gene primers listed in Tab. 1. Relative mRNA expression was determined by a comparative method $\left(2^{-\Delta \Delta^{n} \tau}\right)$ using GAPDH as a reference gene.

\section{Western blotting analysis}

The antibody-based procedures used in this study comply with the recommendations made by the British Journal of Pharmacology (Alexander et al., 2018). The proteins were extracted from mouse liver and HepG2 cells, then equal amounts of protein extract were denatured. After separated by electrophoresis in $12 \%$ SDS-PAGE, the protein samples were transferred onto Polyvinylidene Fluoride (PVDF) membranes. The membranes were blocked with $5 \%$ skimmed milk for $2 \mathrm{~h}$, subsequently incubated with primary antibodies against STAT1 (1:5000), p-STAT1 ${ }^{\mathrm{Y} 701}(1: 1000)$, p-STAT1 $^{\mathrm{S} 727}$ (1:1000), CXCL10 (1:500), p65 (1:1000), pp65 (1:1000), IL-6 (1:1000), TNF $\alpha$ (1:1000), LXR $\alpha$ (1:1000), LXR $\beta$ (1:1000), CHREBP (1:1000), SREBP-1c (1:1000), $\beta$-actin (1:2000) and Lamin B (1:2500) overnight at $4{ }^{\circ} \mathrm{C}$. After three times washing, the membranes were incubated with an HRP-conjugated secondary antibody at room temperature for $2 \mathrm{~h}$. Antibody binding was detected by enhanced chemiluminescence detection kit (Affinity Biosciences, Jiangsu, China) and the digital images were analyzed by Image J. The relative protein levels were normalized to $\beta$-actin or Lamin B.

\section{Data and statistical analysis}

The data and statistical analysis comply with the recommendations on experimental design and analysis in British Journal of Pharmacology (Curtis et al., 2018). Randomization was used to assign samples to the experimental groups and treatment conditions for all in vivo studies. Data collection and acquisition of all in vivo and in vitro experimental paradigms were performed in a blinded manner. All results are 
expressed as the mean \pm SEM $(\mathrm{n}=5)$. Difference among groups was analyzed with one-way analysis of variance (ANOVA) followed by a Bonferroni post hoc analysis using GraphPad Prism 5 software. Post hoc tests were conducted only if $\mathrm{F}$ was significant, and there was no variance inhomogeneity. A value of $\mathrm{p}<0.05$ was considered statistically significant.

\section{Results}

\section{Dios promotes STAT1 and CXCL10 molecular phenotype in livers of NASH mice}

A liver-transcriptome analysis by RNA sequencing (RNA-Seq) was adopted to evaluate the key targets involved in the blockage of NASH disease by Dios. After normalization and analysis of the sequencing data, the DEGs from three sets of the samples were shown in Fig. 1A and 1B (A, HFD vs SD; B, HFD + Dios vs HFD). The DEGs of SD, HFD, and HFD + Dios group were compared with Hierarchical cluster analysis (Fig. 1C) . From the Venn diagram, the result illustrated 456-shared DEGs were discovered between HFD vs SD and HFD + Dios vs HFD (HFD vs SD: 2236 genes; HFD + Dios vs HFD: 566 genes) (Fig. 1D) . To identify the biological features and explore the enrichment-pathways of 456-shared DEGs, the enrichment analysis of GO and KEGG pathway was accomplished by DAVID online tools. The result of GO analysis (Tab. 2) suggested that changes in BP of DEGs were enriched in the immune system process, inflammatory response, chemotaxis, and positive regulation of $\mathrm{T}$ cell activation. Changes in $\mathrm{CC}$ were mainly enriched in the membrane, plasma membrane, and cytoplasm. Changes in MF of DEGs were enriched in GTP binding, chemokine activity, and protein kinase binding. Besides, we found that DEGs were enriched in different pathways, such as antigen processing and presentation, type I diabetes mellitus, cytokine-cytokine receptor interaction, cell adhesion molecules (CAMs) and NF- $\chi \mathrm{B}$ signaling pathway (Tab. 2). PPI network consisting of 408 nodes and 3294 edged (Fig. 1E) was also established by STRING database to reveal the interactive relation of 456 DEGs. The clusters of 456-shared DEGs were examined with MCODE, a plug-in Cytoscape, resulting in four clusters and the most significant cluster scored 32.33 (Fig. 1F) . The top 10 hub genes in the DEGs were confirmed by Cytohubba including TNF, CCL5, CXCL10, STAT1, CXCL9, ITGAX, CD274, IFNG, PSMB8, and CD40 (Fig. 1G). The abbreviations, official names, and functions of these hub genes were shown in Tab. 3 . Ultimately, the qRT-PCR analysis was applied to examine the expression of six genes from DEGs (Fig. 1H) . The levels of those tested genes were aligned with that of RNA-Seq analysis.

\section{Dios reduces the indicators of lipogenesis and inflammation in HepG2 cells}

PA was utilized to establish a NASH model in vitro. The cytotoxicity of PA was measured (Fig. 2A), and there were no apparent changes in HepG2 cells exposed to PA at concentrations of less than 0.2 mM.Fig. 2B and $2 \mathrm{C}$ showed that PA treatment for $24 \mathrm{~h}$ increased intracellular lipid accumulation and TG content in HepG2 cells in a dose-dependent manner. Thus, the concentration of PA at $0.2 \mathrm{mM}$ was used for achieving maximal lipid accumulation without cytotoxicity in experiments. Dios exhibited no cytotoxicity at the lesser dose than $320 \mu \mathrm{M}$ in HepG2 cells (Fig. 2D). Dios dose-dependently enhanced the viability of PA-induced HepG2 cells at a concentration of greater than $5 \mu \mathrm{M}$ (Fig. 2E). Compared with the PA group, both the lipid accumulation and intracellular TG content were reduced significantly by co-treated with Dios and PA in HepG2 cells (Fig. 2F-H) . Dios significantly reduced the expression of STAT1Y701, STAT1S727, and CXCL10 protein after PA treatment (Fig. 2I, J) . Dios also suppressed expressions of the lipogenic proteins LXR $\alpha$, LXR $\beta$, CHREBP, and SREBP-1c (Fig. 2K, L) and decreased that of the inflammatory proteins p-p65 and IL-6 (Fig. 2M, N) induced by PA in HepG2 cells.

Inhibition and overexpression of STAT1 modulate the expressions of relative proteins in HepG2 cells via STAT1/CXCL10 pathway

A STAT1 inhibitor, fludarabine (Fig. S2), was utilized to recognize the role of STAT1 in the hepatoprotec- 
tive effects of Dios. The TG content and lipid accumulation induced by PA were depressed by pretreatment with fludarabine in HepG2 cells (Fig. 3A, B), implying a critical role for STAT1 in the amelioration of hepatocyte steatosis. As shown in Fig. 3C , the inhibition of STAT1 apparently decreased the levels of p-STAT1 ${ }^{\text {Y701 }}$, p-STAT1 ${ }^{\text {S727 }}$, and CXCL10 proteins in HepG2 cells. Additionally, the inhibition of STAT1 significantly reduced the expression of lipogenic proteins $(\operatorname{LXR} \alpha, \operatorname{LXR} \beta$, CHREBP, and SREBP-1c)(Fig. 3D) and downregulated inflammatory indicators p-p65(Fig. 3E) at the protein level. To further confirm our findings, we transfected HepG2 cells with STAT1 overexpression plasmid.Fig. 3F displayed the transfection efficiency of STAT1 overexpression plasmid at the mRNA level. STAT1 overexpression aggravated PA-induced lipid accumulation as observed with O Red O(Fig. 3G) and upregulated the amounts of p-STAT1 ${ }^{\mathrm{Y} 701}$, p-STAT1 ${ }^{\mathrm{S} 727}$, and CXCL10 protein.

\section{Dios attenuates expressions of STAT1 and CXCL10 in HFD-induced NASH mice}

The inhibitory effect of Dios against NASH and the changes of key targets (STAT1 and CXCL10) were studied in HFD-induced NASH mice. As depicted in Fig. 4A, Dios sharply reversed lipid accumulation in livers of HFD-induced NASH mice from Oil Red O staining. Furthermore, as shown in HE staining (Fig. 4B), the liver tissues exhibited histological changes including steatosis, hepatocyte ballooning, and lobular inflammation in the HFD group, but those changes were reduced after Dios treatment. From the hub genes combined with the literature, we selected STAT1 and CXCL10 as key targets for further research. The qRT-PCR result told that Dios dramatically decreased the mRNA levels of STAT1 and CXCL10 induced by HFD (Fig. 4C) . Moreover, the levels of p-STAT1 ${ }^{\mathrm{Y} 701}$, p-STAT1 ${ }^{\mathrm{S} 727}$, and CXCL10 protein were up- and downregulated in the HFD and HFD + Dios group, respectively (Fig. 4D). In the immunofluorescence assay, the nuclear localization of p-STAT1 ${ }^{\text {Tyr701 }}$ (Fig. 4E) and p-STAT1 ${ }^{\text {Ser727 }}$ (Fig. 4F) in the HFD group was as well decreased in the presence of Dios. Correspondingly, the result obtained from the detection of western blot was consistent with that of immunofluorescence(Fig. 4G) . Lipid metabolism played a critical role in the progress of NASH, the expressions of LXR $\alpha$, LXR $\beta$, CHREBP, and SREBP-1c protein relevant with lipogenesis were downregulated after Dios treatment (Fig. 4H). As illustrated in Fig. 4I the amount of p65 phosphorylation and the release of inflammatory factors (TNF $\alpha$ and IL-6) were repressed by Dios.

\section{Inhibition of STAT1 improves functional parameters and liver morphological in HFD-induced NASH mice}

To investigate the contribution of STAT1 in the protective effect of Dios on NASH, STAT1 inhibitor fludarabine was pretreated to induce low expression of STAT1 in mice. A substantial body-weight reduction was found in NASH mice treated with Dios and fludarabine(Fig. 5A, B), with no effect on food consumption was found among all groups (Fig. S3A, B). Fig. 5C showed that Dios and fludarabine markedly alleviated and even reverse pathological changes in liver morphology of NASH mice. Additionally, STAT1-inhibited mice treated with Dios exhibited a decreased index of the livers(Fig. 5D) and abdominal fat pad, without a significant difference in the ration of kidney and spleen (Fig. S3C, D) . The results of Oil Red O staining and the contents of liver TG and TC revealed that there was massive lipid accumulation in the livers of NASH mice, which was fully alleviated by Dios in mice and STAT1-inhibited mice (Fig. 5E-H) . Furthermore, the results of the biochemical analysis showed that Dios also reduced the levels of serum TC, LDL-C, ALT, and AST, but no difference was seen for serum TG and serum HDL-C(Fig. 5I-N) . Meanwhile, the obvious pathological changes of livers were observed including increased hepatic steatosis, hepatocyte ballooning, and lobular inflammation, but no fibrosis. These changes were remarkably ameliorated by Dios and fludarabine in HFD-fed mice(Fig. 5O, P) . SAF score was performed by histological analysis according to a grading system established for mouse models of NAFLD/NASH, showing that the majority of HFD-fed mice displayed a pathophysiological characteristic of NASH, whereas both Dios and fludarabine dramatically attenuated that characteristic (Fig. S4A-D) . 


\section{Interference with STAT1/CXCL10 pathway mediates Dios-conferred hepatopro- tective effect}

STAT1/CXCL10 was regarded as a crucial pathway relevant to lipogenesis and inflammation in NASH mice. Fig. 6A revealed that the levels of p-STAT1 ${ }^{\mathrm{Y} 701}$, p-STAT1 ${ }^{\mathrm{S} 727}$, and CXCL10 protein significantly were eliminated in the HFD + Dios group. Additionally, in comparison with the HFD group, the expressions of these proteins were inhibited in the HFD-treated group with the STAT1 inhibitor. As depicted in Fig. 6B, C , Dios-treated mice displayed decreased protein expression levels of LXR $\alpha$, LXR $\beta$, CHREBP, SREBP-1c, and p-p65. The protein levels of all of these markers were completely normalized in the fludarabine-treated mice. Because STAT1 was reported to be an upstream regulator of CXCL10, siCXCL10 was used to further investigate the relation between them. Fig. 6D showed the transfection efficiency of CXCL10 siRNA at the mRNA level. Compared with siNull treatment, siCXCL10-treated HepG2 cells displayed down-regulated expression levels of CXCL10, LXR $\alpha, \mathrm{LXR} \beta$, and p-p65 proteins, but no effects on the levels of STAT1, p-STAT1 ${ }^{\mathrm{Y} 701}$, and p-STAT1 ${ }^{\mathrm{S} 727}$ protein $($ Fig. $\mathbf{6 E}, \mathbf{F})$, which indicated that CXCL10 was the downstream targeted gene of STAT1 and could regulate lipogenesis and inflammatory response by LXR $\alpha / \beta$ and p65, respectively.

\section{Discussion}

Emerging evidence suggested that histopathological evaluation of biopsy specimens is a gold standard for diagnosing NASH (Brown \& Kleiner, 2016). The typical characteristic such as steatosis, lobular inflammation, and hepatocellular ballooning are all observed in NASH model liver but fibrosis is not uncertain (Takahashi \& Fukusato, 2014). Actually, by Oil Red O, HE, and Mason trichrome staining in the livers of NASH mice in our study, liver tissues occurred these histological changes without fibrosis. Meanwhile, the SAF scores of liver histology assisted us to recognize that the establishment of NASH mice model was successful (Bedossa \& Consortium, 2014). As for the impact of Dios on NASH mice, Dios distinctly blocked the pathological changes of liver and diminished the body weight, liver index, the content of liver TG and TC, and the serum levels of TC, LDL-C, ALT, and AST. In vitro HepG2 cells, Dios eliminated lipid deposition and TG content induced by PA. Our finding was consistent with Zeng's report (Zeng et al., 2017). The primary study showed that Dios exerted a hepatoprotective effect against NASH, but the key target genes and proteins interfered by Dios in NASH mice were unclear and should be explored. Hence, the RNA-Seq analysis was employed in the further seeking of the novel key targets.

Here a total of 456 mutual DEGs were identified using liver RNA-Seq in our experiment. After the enrichment analysis of those DEGs, most of them were focused on the fields of the inflammatory and immune response. Especially, STAT1 and CXCL10 were the hub genes among the DEGs and were likely the major regulated targets for Dios. In the liver of NASH mice and patients, the levels of STAT1 phosphorylation and CXCL10 were increased (Chmielewski et al., 2014; Zhang et al., 2014). Similarly, the mRNA and protein expressions of STAT1 and CXCL10 were significantly upregulated both in NASH mice and HepG2 cells, however, Dios exhibited the repression on those expressions. Our results indicated that Dios reduced the nuclear localization of p-STAT1 ${ }^{\mathrm{Y} 701}$ and p-STAT1 ${ }^{\mathrm{S} 727}$ in livers of NASH mice induced by HFD. In terms of the related reference, saturated free fatty acids induced hepatocyte lipotoxicity in NASH could cause the nuclear localization of pSTAT1 ${ }^{\mathrm{Y} 701}$, and this conclusion supported our results appropriately (Tomita et al., 2017). In a word, STAT1 and CXCL10 considered as key targets had a pivotal role in the attenuation of Dios on NASH. However, STAT1 and CXCL10 mediated related proteins involved in the pathways of lipogenesis and inflammatory response needed to be discussed. Moreover, the hepatoprotective mechanism of Dios against NASH through STAT1 / CXCL10 pathway was also worth elucidating.

NASH displayed the disorder in lipid metabolism and inflammation (Farrell et al., 2012), while the elevation of STAT1 and CXCL10 expression contributed to that disorder (Neville, Mathiak \& Bagasra, 1997; Sisler et al., 2015). In other words, if the disorder were mediated by STAT1 and CXCL10, the down-stream proteins involved in the pathways of lipogenesis and inflammation should be explored. In the pathway of lipogenesis, 
LXR $\alpha / \beta$, SREBP-1c, and ChREBP were the important transcription factors to regulate hepatic lipogenesis in NAFLD (Ahmed \& Byrne, 2007; Scorletti \& Byrne, 2018), and LXR $\alpha / \beta$ could activate SREBP-1c and ChREBP to mediate the metabolism of cholesterol and fatty acids (Fan et al., 2017; Higuchi et al., 2008). Our results claimed that when STAT1 and CXCL10 were suppressed by Dios, the levels of LXR $\alpha / \beta$, ChREBP, and SREBP-1c were also decreased in HepG2 cells and the liver of NASH mice. In the pathway of inflammation, the important marker NF- $\chi \mathrm{B}$ (p65), and the inflammatory factors including TNF $\alpha$ and IL-6, were regarded as the indicator of hepatic inflammation (Pea et al., 2005; Tak \& Firestein, 2001). All these indicators were inhibited by Dios as well after the mediation of STAT1 and CXCL10.

For better identification of the relation between the downstream proteins and the STAT1/ CXCL10 pathway, the STAT1-inhibitor, overexpression vector of STAT1, and CXCL10 siRNA were used here. The pretreatment of STAT1-inhibitor fludarabine downregulated the level of STAT1 phosphorylation, and induced the elimination of relative protein levels of lipogenesis and inflammatory response in HepG2 cells and NASH mice, as well as restrained the changes of biochemistry indicators and hepatic pathological parameters. According to the reference, lipid accumulation and hepatic TG level increased in the livers of STAT1 $1^{+/+}$ mice (Sisler et al., 2015). In our result, the hepatocyte steatosis induced by PA was aggravated due to the over-expression of STAT1, but that was overwhelmed by Dios. Combined with our study and the numerous evidence, the expression of CXCL10 was positively correlated with the STAT1 phosphorylation (Burke et al., 2013; Tomita et al., 2017). The inhibition or over-expression of STAT1 led to a decrease or increase of CXCL10 protein expression. Meanwhile, the lipogenic factor LXR $\alpha / \beta$ and inflammatory protein NF- $x \mathrm{~B}$ (p65) were diminished in CXCL10-deficient mice (Zhang et al., 2014). Our finding equally manifested that when HepG2 cells were administrated with CXCL10 siRNA, the expressions of LXR $\alpha / \beta$ and p-p65 protein were reduced. However, the levels of STAT1 and p-STAT1 after siCXCL10 treatment in HepG2 cells showed no differences in our result. The whole potential hepatoprotective mechanism of Dios against NASH was revealed at the same time. Dios modulated lipogenesis and inflammatory response of NASH were most likely through the STAT1/CXCL10 signaling pathway.

Taken together, the hepatoprotective mechanism of Dios against NASH was through the modulation of lipogenesis and the alleviation of inflammatory response via STAT1/CXCL10-dependent pathway. Dios has good hepatoprotective effects and potentiality as a promising therapeutic agent for NASH in clinical application.

\section{Author contributions}

B. Z. designed the research. N. L. performed the experiments, with the assistance of C. Y. and Y. Z. analyzed the data. N. L. wrote the initial manuscript and B. Z. revised the manuscript.

\section{References}

Ahmed MH, \& Byrne CD (2007). Modulation of sterol regulatory element binding proteins (SREBPs) as potential treatments for non-alcoholic fatty liver disease (NAFLD). Drug Discov Today 12: 740-747.

Alexander SPH, Roberts RE, Broughton BRS, Sobey CG, George CH, Stanford SC, et al. (2018). Goals and practicalities of immunoblotting and immunohistochemistry: A guide for submission to the British Journal of Pharmacology. Brit J of pharmacol 175: 407-411.

Bedossa P, \& Consortium FP (2014). Utility and appropriateness of the fatty liver inhibition of progression (FLIP) algorithm and steatosis, activity, and fibrosis (SAF) score in the evaluation of biopsies of nonalcoholic fatty liver disease. Hepatology 60: 565-575. 
Brown GT, \& Kleiner DE (2016). Histopathology of nonalcoholic fatty liver disease and nonalcoholic steatohepatitis. Metabolism 65:1080-1086.

Burke SJ, Goff MR, Lu D, Proud D, Karlstad MD, \& Collier JJ (2013). Synergistic Expression of the CXCL10 Gene in Response to IL-1 $\beta$ and IFN- $\gamma$ Involves NF- $x$ B, Phosphorylation of STAT1 at Tyr701, and Acetylation of Histones H3 and H4. J Immunol 191: 323-336.

Chmielewski S, Olejnik A, Sikorski K, Pelisek J, Blaszczyk K, Aoqui C, et al. (2014). STAT1-dependent signal integration between IFNgamma and TLR4 in vascular cells reflect pro-atherogenic responses in human atherosclerosis. PLoS One 9: e113318.

Curtis MJ, Alexander S, Cirino G, Docherty JR, George CH, Giembycz MA, et al. (2018). Experimental design and analysis and their reporting II: updated and simplified guidance for authors and peer reviewers. Brit J of pharmacol 175: 987-993.

Fan Q, Norgaard RC, Bindesboll C, Lucas C, Dalen KT, Babaie E, et al. (2017). LXRalpha Regulates Hepatic ChREBPalpha Activity and Lipogenesis upon Glucose, but Not Fructose Feeding in Mice. Nutrients 9 .

Farrell GC, van Rooyen D, Gan L, \& Chitturi S (2012). NASH is an Inflammatory Disorder: Pathogenic, Prognostic and Therapeutic Implications. Gut Liver 6: 149-171.

Grohmann M, Wiede F, Dodd GT, Gurzov EN, Ooi GJ, Butt T, et al.(2018). Obesity Drives STAT-1Dependent NASH and STAT-3-Dependent HCC. Cell 175: 1289-1306.e1220.

Herzig S, \& Shaw RJ (2018). AMPK: guardian of metabolism and mitochondrial homeostasis. Nat Rev Mol Cell Biol 19: 121-135.

Higuchi N, Kato M, Shundo Y, Tajiri H, Tanaka M, Yamashita N, et al. (2008). Liver X receptor in cooperation with SREBP-1c is a major lipid synthesis regulator in nonalcoholic fatty liver disease. Hepatol Res 38: 1122-1129.

Huang DW, Sherman BT, \& Lempicki RA (2009). Bioinformatics enrichment tools: paths toward the comprehensive functional analysis of large gene lists. Nucleic Acids Res 37: 1-13.

Ibrahim SH, Hirsova P, Tomita K, Bronk SF, Werneburg NW, Harrison SA, et al. (2016). Mixed lineage kinase 3 mediates release of C-X-C motif ligand 10-bearing chemotactic extracellular vesicles from lipotoxic hepatocytes. Hepatology 63: 731-744.

Kilkenny C, Browne W, Cuthill I, Emerson M, \& Altman D (2010). Animal research: reporting in vivo experiments: the ARRIVE guidelines. Brit J of pharmacol 160: 1577-1579.

Lau JK, Zhang X, \& Yu J (2017). Animal models of non-alcoholic fatty liver disease: current perspectives and recent advances. J Pathol 241: 36-44.

Liao W, Ning Z, Chen L, Wei Q, Yuan E, Yang J, et al. (2014). Intracellular antioxidant detoxifying effects of diosmetin on 2,2-azobis(2-amidinopropane) dihydrochloride (AAPH)-induced oxidative stress through inhibition of reactive oxygen species generation. J Agric Food Chem 62: 8648-8654.

Liu JF, Ma Y, Wang Y, Du ZY, Shen JK, \& Peng HL (2011). Reduction of lipid accumulation in HepG2 cells by luteolin is associated with activation of AMPK and mitigation of oxidative stress. Phytother Res 25: $588-596$.

Liu Q, Ci X, Wen Z, \& Peng L (2018). Diosmetin Alleviates Lipopolysaccharide-Induced Acute Lung Injury through Activating the Nrf2 Pathway and Inhibiting the NLRP3 Inflammasome. Biomol Ther (Seoul) 26: 157-166.

Marcolin E, San-Miguel B, Vallejo D, Tieppo J, Marroni N, Gonzalez-Gallego J, et al. (2012). Quercetin treatment ameliorates inflammation and fibrosis in mice with nonalcoholic steatohepatitis. J Nutr 142: 
1821-1828.

Neville LF, Mathiak G, \& Bagasra O (1997). The immunobiology of interferon-gamma inducible protein 10 $\mathrm{kD}$ (IP-10): a novel, pleiotropic member of the C-X-C chemokine superfamily. Cytokine Growth Factor Rev 8: 207-219.

Pea Ad, Leclercq I, Field J, George J, Jones B, \& Farrell G (2005). NF-xB Activation, Rather Than TNF, Mediates Hepatic Inflammation in a Murine Dietary Model of Steatohepatitis. Gastroenterology 129:16631674 .

Schuster S, Cabrera D, Arrese M, \& Feldstein AE (2018). Triggering and resolution of inflammation in NASH. Nat Rev Gastroenterol Hepatol 15: 349-364.

Scorletti E, \& Byrne CD (2018). Omega-3 fatty acids and non-alcoholic fatty liver disease: Evidence of efficacy and mechanism of action. Mol Aspects Med 64: 135-146.

Sisler J, Morgan M, Raje V, Grande R, Derecka M, Meier J, et al.(2015). The Signal Transducer and Activator of Transcription 1 (STAT1) Inhibits Mitochondrial Biogenesis in Liver and Fatty Acid Oxidation in Adipocytes. PLoS One 10: e0144444.

Strzyz P (2020). AMPK against NASH. Nat Rev Mol Cell Biol 21(4).

Tak PP, \& Firestein GS (2001). NF-kappaB: a key role in inflammatory diseases. J Clin Invest 107: 7-11.

Takahashi Y, \& Fukusato T (2014). Histopathology of nonalcoholic fatty liver disease/nonalcoholic steatohepatitis. World J Gastroenterol 20: 15539-15548.

Tomita K, Freeman BL, Bronk SF, LeBrasseur NK, White TA, Hirsova P, et al. (2016). CXCL10-Mediates Macrophage, but not Other Innate Immune Cells-Associated Inflammation in Murine Nonalcoholic Steatohepatitis. Sci Rep 6: 28786.

Tomita K, Kabashima A, Freeman BL, Bronk SF, Hirsova P, \& Ibrahim SH (2017). Mixed Lineage Kinase 3 Mediates the Induction of CXCL10 by a STAT1-Dependent Mechanism During Hepatocyte Lipotoxicity. J Cell Biochem 118: 3249-3259.

Wang LF, Wang XN, Huang CC, Hu L, Xiao YF, Guan XH, et al.(2017). Inhibition of NAMPT aggravates high fat diet-induced hepatic steatosis in mice through regulating Sirt1/AMPK $\alpha /$ SREBP1 signaling pathway. Lipids Health Dis 16.

Wu L, Wang Y, Chi G, Shen B, Tian Y, Li Z, et al. (2019). Morin reduces inflammatory responses and alleviates lipid accumulation in hepatocytes. J Cell Physiol 234: 19785-19798.

Yang Y, Gong XB, Huang LG, Wang ZX, Wan RZ, Zhang P, et al.(2017). Diosmetin exerts anti-oxidative, anti-inflammatory and anti-apoptotic effects to protect against endotoxin-induced acute hepatic failure in mice. Oncotarget 8: 30723-30733.

Younossi Z, Anstee QM, Marietti M, Hardy T, Henry L, Eslam M, et al. (2018). Global burden of NAFLD and NASH: trends, predictions, risk factors and prevention. Nat Rev Gastroenterol Hepatol 15:11-20.

Zeng X, Yang J, Hu O, Huang J, Ran L, Chen M, et al. (2017). Dihydromyricetin Ameliorates Nonalcoholic Fatty Liver Disease by Improving Mitochondrial Respiratory Capacity and Redox Homeostasis Through Modulation of SIRT3 Signaling. Antioxid Redox Signal 30: 163-183.

Zhang X, Shen J, Man K, Chu ES, Yau TO, Sung JC, et al. (2014). CXCL10 plays a key role as an inflammatory mediator and a non-invasive biomarker of non-alcoholic steatohepatitis. J Hepatol 61:13651375 .

Zheng FF, Guo XM, Zhong BL, \& Jiang XY (2018). The protective effect of diosmetin on non-alcoholic fatty liver disease of young rats. Chinese Journal of Clinical Anatomy 36: 520-526. 
Zhou R, Yi L, Ye X, Zeng X, Liu K, Qin Y, et al. (2018). Resveratrol Ameliorates Lipid Droplet Accumulation in Liver Through a SIRT1/ ATF6-Dependent Mechanism. Cell Physiol Biochem 51:2397-2420.

\section{Tables}

Table 1 Primers used for real-time RT-PCR analysis.

Table 2 GO and KEGG pathway enrichment analysis of DEGs.

Table 3 Functional roles of 10 hub genes.

\section{Figure Legends}

Figure 1 Effect of Dios on molecular phenotype of STAT1 and CXCL10 in mice liver tissues of NASH by using RNA-Seq. (A-B) Identification of DEGs in different groups. (A) HFD vs SD group. (B) HFD + Dios vs HFD group. The red points represent upregulated genes. The green points represent downregulated genes. The blue points represent genes with no significant difference. DEGs were screened on $\mathrm{P}<0.05$ and $\mid \log _{2}$ (Fold change) $\mid>1$. (C) Heat map analysis was employed to the discrimination of expression profile of DEGs across the samples. Red and blue areas separately represent highly and lowly expressed genes in mice livers among SD, HFD and HFD + Dios groups. (D) Venn diagram of DEGs among different groups. 456-shared DEGs had obtained from this diagram. (E) The PPI network of 456-shared DEGs was analyzed through STING database. There were 408 nodes and 3294 edged in the PPI network. (F) The significant modules of DEGs. The significant modules obtained from PPI network of DEGs were identified via MCODE. The investigated scores of cluster 1 to 4 were 32.33, 18.00, 10.94 and 9.81, respectively. (G) The ten hub genes including STAT1 and CXCL10 were confirmed by Cytohubba. (H) The amount of DEGs mRNA expression (FPR2, PSMB8, PTAFR, CCR7, CXCR4, LCK) was measured under the method of quantitative RT-PCR. Data are expressed as mean \pm SEM $(\mathrm{n}=5)$. ${ }^{*} \mathrm{P}<0.05$, vs. the $\mathrm{SD}$ group; ${ }^{\#} \mathrm{P}$ $<0.05$, vs. the HFD group. DEGs, different expression genes; SD, standard diet; HFD, high fat diet; Dios, Diosmetin; PPI, protein-protein interaction; MCODE, molecular complex detection.

Figure 2 Impact of Dios on the indicators of lipogenesis and inflammation induced by PA in HepG2 cells. (A) HepG2 cells viabilities were measured with MTT assay after PA treatment under different concentrations $(0.10,0.15,0.20,0.25,0.30,0.35$, or $0.4 \mathrm{mM})$ for $24 \mathrm{~h}$. (B) Representative images of hepatocellular lipid accumulation by Oil Red O staining. Scale bar, $20 \mu \mathrm{m}$. (C) Intracellular TG content of PA-induced HepG2 cells was detected using commercial kit. (D) HepG2 cells viabilities were measured with MTT assay after Dios treatment under different concentrations $(5,10,20,40,80,160$, and $320 \mu \mathrm{M})$ for $24 \mathrm{~h}$. (E) HepG2 cells viabilities were measured with MTT after $0.2 \mathrm{mM}$ PA treatment in the presence or absence of Dios for $24 \mathrm{~h}$. (F) Representative images of hepatocellular lipid accumulation which were treated with $0.2 \mathrm{mM}$ PA in the presence or absence of Dios by Oil Red O staining. Scale bar, $20 \mu \mathrm{m}$. (G) Intracellular TG content of was detected by using commercial kit after the treatment of $0.2 \mathrm{mM}$ PA in the presence or absence of Dios. (H) Representative fluorescence of HepG2 cells was shown after PA and Dios treatment with Nile red staining, and the mean fluorescent intensity was measured as well. Scale bar, $20 \mu \mathrm{m}$. (I-J) The levels of STAT1, p-STAT1 ${ }^{\mathrm{Y} 701}$, p-STAT1 ${ }^{\mathrm{S} 727}$ and CXCL10 protein expression in HepG2 Cells after the treatment of $0.2 \mathrm{mM}$ PA in the presence or absence of Dios for $24 \mathrm{~h}$. (K-N) The levels of LXR $\alpha, \operatorname{LXR} \beta$, CHREBP, SREBP-1c, IL-6, p65 and p-p65 protein expression after the same treatment as above (I-J). Data are presented as mean $\pm \mathrm{SEM}(\mathrm{n}=5)$. ${ }^{*} \mathrm{P}<0.05$, vs. the Control group; ${ }^{\#} \mathrm{P}<0.05$, vs. the PA group. TG, triglyceride; PA, palmitic acid.

Figure 3 Inhibition and overexpression of STAT1 modulate the expressions of relative proteins in HepG2 cells via STAT1/CXCL10 pathway. From A to E, HepG2 cells pretreated with or without fludarabine inhibitor $(10 \mu \mathrm{M})$ before the stimulation of Dios and PA. (A) Intracellular TG content of HepG2 
cells after treatment. (B) Lipid accumulation by Oil Red O staining. The expression of (C) STAT1, pSTAT1 $^{\text {Y701, }}$ p-STAT1 ${ }^{\text {S727 }}$, CXCL10, (D) LXR $\alpha$, LXR $\beta$, CHREBP and SREBP-1c (E) p65, p-p65 were examined by Western blot assay and quantified by densitometric analysis. $\beta$-actin was used as an internal control. From F to H, HepG2 cells treated with plasmid either pcDNA3.1 or STAT1-pcDNA3.1 for 24h before the stimulation of Dios and PA. (F) The mRNA expression of STAT1. (G) Lipid accumulation by Oil Red O staining. $(\mathrm{H})$ The levels of STAT1, p-STAT1 ${ }^{\mathrm{Y} 701}$, p-STAT1 ${ }^{\mathrm{S} 727}$ and CXCL10 protein, and $\beta$-actin was used as an internal control. Data are presented as mean \pm SEM $(\mathrm{n}=5)$. ${ }^{*} \mathrm{P}<0.05$, compared between the marked groups. Flu, fludarabine.

Figure 4 2. Effect of Dios on the expressions of STAT1 and CXCL10 in HFD-induced NASH mice. (A, B) Histological evaluations of mice liver tissues in different groups were through Oil Red $\mathrm{O}$ staining and HE staining, respectively. Scale bar $100 \mu \mathrm{m}$. (1, steatosis; 2, hepatocyte ballooning; 3, lobular inflammation). (C) The mRNA expressions of STAT1 and CXCL10 in mice livers by qRT-PCR assay. (D) The expression of total STAT1, p-STAT1 ${ }^{\mathrm{Y} 701}$, p-STAT1 ${ }^{\mathrm{S} 727}$ and CXCL10 proteins were examined through western blot assay. $\beta$-actin was used as an internal control. (E, F) Representative immunofluorescence of mice liver sections were stained with (E) phospho-STAT1 (Tyr701) and (F) phospho-STAT1 (Ser727) antibodies. The staining was observed following the addition of DAPI by fluorescence microscope. Scale bar, 20um. (G) The levels of p-STAT1 ${ }^{\mathrm{Y} 701}$, p-STAT1 ${ }^{\mathrm{S} 727}$, STAT1, $\beta$-actin and LaminB protein expression from the cytosol into the nucleus were analyzed by Western blot. $(\mathrm{H})$ The determination of relative proteins expression of lipid metabolism were used Western blot. (I) The expression of IL-6, TNF $\alpha$, p65 and p-p65 proteins. $\beta$-actin was used as an internal control. Data are expressed as mean \pm SEM $(\mathrm{n}=5) .{ }^{*} \mathrm{P}<0.05$, vs. the SD group; ${ }^{\#} \mathrm{P}<0.05$, vs. the HFD group. p-STAT1 ${ }^{\mathrm{Y} 701}$, phospho-STAT1 (Tyr701); p-STAT1 ${ }^{\mathrm{S} 727}$, phospho-STAT1 (Ser727).

Figure 5 Inhibition of STAT1 improves functional parameters and liver morphological in HFDinduced NASH mice. (A-B) Weekly body weights of mice (A) and STAT1-inhibited mice (B) were recorded during 18 weeks. (C) Gross examination of liver. (D) Liver index. (E) Histological analysis of mice livers by Oil Red O staining Scale bars, $200 \mu \mathrm{m}$ (top panel), $100 \mu \mathrm{m}$ (middle panel) and $50 \mu \mathrm{m}$ (lower panel). (F) Percentage of Oil Red O staining area in mice livers quantified by Image J. The contents of liver TG (G) and TC $(\mathrm{H})$ were detected using a commercial kit. The levels of serum TG (I), TC (J), LDL-C (K), HDL-C $(\mathrm{L})$, ALT (M) and AST (N) were tested via corresponding kits. (O, P) Histological changes of liver sections was respectively evaluated with hematoxylin eosin staining and mason trichrome staining. Scale bar, 100 $\mu \mathrm{m}$. Data are presented as mean \pm SEM $(\mathrm{n}=5) .{ }^{*} \mathrm{P}<0.05$, compared between the marked groups. TC, total cholesterol; LDL-C, low density lipoprotein cholesterol; HDL-C, high density lipoprotein cholesterol; ALT, alanine aminotransferase; AST, aspartate aminotransferase.

Figure 6 Interference with STAT1/CXCL10 pathway mediates Dios-conferred hepatoprotective effect. (A-C) The levels of p-STAT1 ${ }^{\mathrm{Y} 701}$, p-STAT1 ${ }^{\mathrm{S} 727}$, STAT1, CXCL10, LXR $\alpha$, LXR $\beta$, CHREBP, SREBP-1C, p65 and p-p65 protein in STAT1-inhibited or non-inhibited mice. (D) The mRNA expression of CXCL10 in HepG2 cells transfected with siCXCL10. (E, F)The amount of STAT1, pSTAT1 ${ }^{\text {Y701, }}$ pSTAT1 $^{\mathrm{S} 727}$, CXCL10, LXR $\alpha, \mathrm{LXR} \beta$, p65, and p-p65 expression, and $\beta$-actin was used as an internal control. Data are presented as mean \pm SEM $(\mathrm{n}=5)$. ${ }^{*} \mathrm{P}<0.05$, compared between the marked groups.

\section{Hosted file}

Tables.docx available at https://authorea.com/users/348842/articles/474038-diosmetinmodulates-lipogenesis-and-alleviates-inflammatory-response-in-nonalcoholicsteatohepatitis-through-stat1-cxcl10-dependent-pathway

\section{Hosted file}

Figures.docx available at https://authorea.com/users/348842/articles/474038-diosmetinmodulates-lipogenesis-and-alleviates-inflammatory-response-in-nonalcoholic-

steatohepatitis-through-stat1-cxcl10-dependent-pathway 